\title{
Minimizing Variability of Cascade Impaction Measurements in Inhalers and Nebulizers
}

\author{
Matthew Bonam, ${ }^{1}$ David Christopher, ${ }^{2}$ David Cipolla, ${ }^{3}$ Brent Donovan, ${ }^{4}$ David Goodwin, ${ }^{5}$ Susan Holmes, ${ }^{6}$ \\ Svetlana Lyapustina, ${ }^{7,17}$ Jolyon Mitchell, ${ }^{8}$ Steve Nichols, ${ }^{5}$ Gunilla Pettersson, ${ }^{9}$ Chris Quale, ${ }^{10}$ Nagaraja Rao, ${ }^{11}$ \\ Dilraj Singh, ${ }^{12}$ Terrence Tougas, ${ }^{13}$ Mike Van Oort, ${ }^{14}$ Bernd Walther, ${ }^{15}$ and Bruce Wyka ${ }^{16}$
}

Received 23 August 2007; accepted 19 January 2008; published online 28 February 2008

\begin{abstract}
The purpose of this article is to catalogue in a systematic way the available information about factors that may influence the outcome and variability of cascade impactor (CI) measurements of pharmaceutical aerosols for inhalation, such as those obtained from metered dose inhalers (MDIs), dry powder inhalers (DPIs) or products for nebulization; and to suggest ways to minimize the influence of such factors. To accomplish this task, the authors constructed a cause-and-effect Ishikawa diagram for a $\mathrm{CI}$ measurement and considered the influence of each root cause based on industry experience and thorough literature review. The results illustrate the intricate network of underlying causes of CI variability, with the potential for several multi-way statistical interactions. It was also found that significantly more quantitative information exists about impactor-related causes than about operatorderived influences, the contribution of drug assay methodology and product-related causes, suggesting a need for further research in those areas. The understanding and awareness of all these factors should aid in the development of optimized CI methods and appropriate quality control measures for aerodynamic particle size distribution (APSD) of pharmaceutical aerosols, in line with the current regulatory initiatives involving quality-by-design (QbD).
\end{abstract}

KEY WORDS: aerosol; impactor; inhaler; nebulizer; variability.

\section{INTRODUCTION}

Quality control systems, generally speaking, are intended (a) to monitor (i.e., measure) selected parameters of a product, which are deemed critical quality attributes, (b) to compare outcomes of these measurements with pre-determined sets of

Editorial Comment: The International Pharmaceutical Aerosol Consortium on Regulation and Science (IPAC-RS) is an international association of innovator and generic companies that develop, manufacture or market orally inhaled and nasal drug products for local and systemic treatment of a variety of debilitating diseases such as asthma, chronic obstructive pulmonary disease and diabetes. IPAC-RS is committed to advancing consensus-based, scientifically driven standards and regulations for these products, with the purpose of facilitating the availability of high-quality, safe, and efficacious drug products to patients.

\footnotetext{
${ }^{1}$ Analytical Development, AstraZeneca Charnwood, Loughborough, Leicestershire, UK.

${ }^{2}$ Statistics, Schering Plough Research Institute, Kenilworth, New Jersey, USA.

${ }^{3}$ Pharmaceutical Sciences, Aradigm, Hayward, California, USA.

${ }^{4}$ Schering-Plough Research Institute, Union, New Jersey, USA.

${ }^{5}$ Sanofi-Aventis, Holmes Chapel, UK.

${ }^{6}$ Global Regulatory Affairs, GlaxoSmithKline, Research Triangle Park, North Carolina, USA.

${ }^{7}$ Pharmaceutical Practice, Drinker Biddle \& Reath LLP, 1500 K Street, N.W., Suite 1100, Washington, District of Columbia 20005-1209, USA

${ }^{8}$ Trudell Medical International, London, Ontario, Canada.

${ }^{9}$ Analytical Development, AstraZeneca R\&D, Lund, Sweden.
}

criteria deemed to describe "typical", "in-control" situations, and (c) to alert the manufacturer if any of the monitored metrics are approaching or exceeding their allowed ranges. In order to fulfill these requirements, an appropriate quality control system must have at its disposal such tools and metrics that would allow the manufacturer to extract information about the product itself,

${ }^{10}$ Novo Nordisk Delivery Technologies, Inc., Hayward, California, USA ${ }^{11}$ Aerosol Drug Delivery, Nektar Therapeutics, San Carlos, California, USA.

${ }^{12}$ Novartis Pharma AG, Basel, Switzerland.

${ }^{13}$ Boehringer Ingelheim, Ridgefield, Connecticut, USA.

${ }^{14}$ Inhaled Product Development, GlaxoSmithKline, Research Triangle Park, North Carolina, USA.

${ }^{15}$ Drug Delivery, Boehringer Ingelheim Pharma GmbH \& Co. KG, Biberach, Germany.

${ }^{16}$ Oral and Respiratory Product Development, Schering-Plough Research Institute, Summit, New Jersey, USA.

${ }^{17}$ To whom correspondence should be addressed. (e-mail: svetlana. lyapustina@dbr.com)

NOTATIONS: (A)CI, (Andersen) cascade impactor; API, active pharmaceutical ingredient; APSD, aerodynamic particle size distribution; DPI, dry powder inhaler; ECD, effective cut-off diameter; IP, induction port; IPAC-RS, International Pharmaceutical Aerosol Consortium on Regulation and Science; LOD, limit of detection; LOQ, limit of quantitation; MB, mass balance (obtained from a cascade impactor measurement); MDI, pressurized metered dose inhaler; MSLI, multi-stage liquid impinger; NGI, next generation pharmaceutical impactor; $\mathrm{QbD}$, quality by design. 
unobscured by potential contributions from the measurement method. Unfortunately, one of the core measurements of an important quality parameter for orally inhaled and nasal drug products, namely a cascade impactor measurement of aerodynamic particle size distribution (APSD), while providing information not always available from other particle-sizing methods (1-6) has long been recognized in the scientific community as being problematic because many factors not related to product quality may influence the CI measurement outcome (2,7-8). This report aims to catalogue in a systematic way all pertinent information about factors potentially influencing a CI measurement outcome and to suggest ways to minimize CI method-related uncertainties and optimize the CI method for a given application. This review will be of particular help when a company is developing a CI method in a systematic way, identifying various sources of variability as well as ways to eliminate, minimize and/or control them. Furthermore, this paper could serve as an aid in identifying root-causes of "deviating" CI results during laboratory investigations, either as part of method development or in routine quality control.

More generally, the current consolidated review of factors influencing CI measurements could serve as a step towards development of improved approaches of controlling
APSD in inhaled products and should help individual sponsor discussions with regulators under the current $\mathrm{QbD}$ paradigm (9-13).

The other aspect of the QbD approach as it relates to CI and APSD measurements, namely establishing links between quality controls and clinical outcomes, has been discussed elsewhere (14) and is outside the scope of this review.

\section{METHODS}

A working group of the International Pharmaceutical Aerosol Consortium on Regulation and Science (IPAC-RS) was formed in 2005 to explore the issue of CI method variability. The group included experts in product development, manufacturing, quality control and regulatory affairs. Based on the members' experience and thorough literature review, the group constructed an Ishikawa Diagram (Fig. 1) that visualized and categorized the factors that may affect a CI measurement. As is typical for Ishikawa Diagrams, the following major categories of factors were included: "man" (i.e., CI operator/analyst), "machine" (i.e., the CI, its accessories and operating/environmental conditions), "mea-

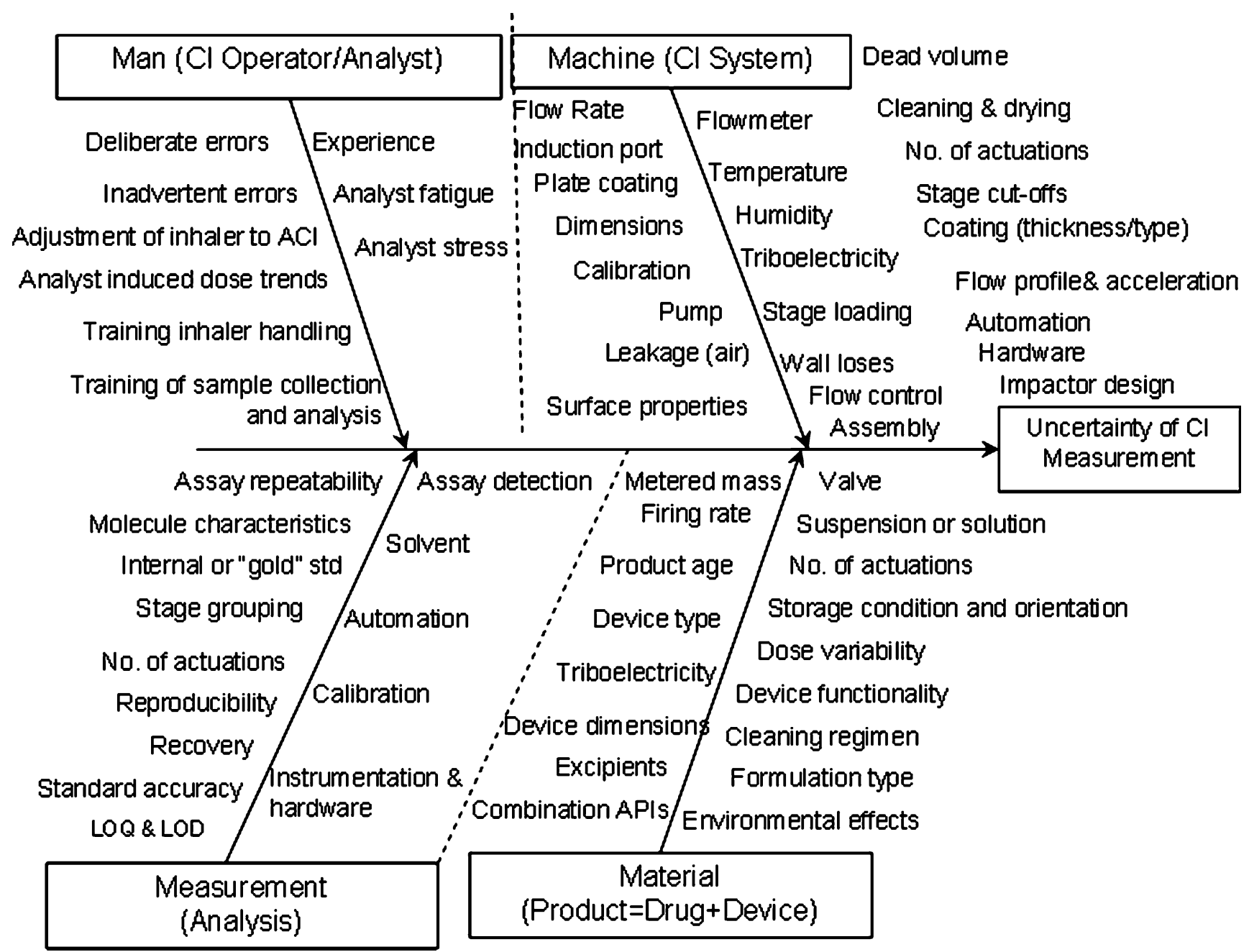

Fig. 1. An Ishikawa Diagram summarizing factors influencing the uncertainty of a CI measurement (both its bias, or systematic error, and variability, or random error) 
surement" [e.g., detection and assay of the active pharmaceutical ingredient (API), such as HPLC analysis], and "material" (i.e., medicinal formulation together with its delivery device). Focused literature searches and internal company surveys were then undertaken to assemble available evidence in each category and to provide specific recommendations for minimizing influence of the identified factors.

\section{RESULTS AND DISCUSSION}

\section{Man (CI Operator/Analyst)}

Performing a CI measurement requires several manual operations, such as impactor assembly, connections to vacuum valves and pump, adjustment of air flowrate, inhaler manipulation and actuation, quantitative recovery of the deposited API, dilution for subsequent HPLC-spectrophotometric assay of API or other type of analysis. Human involvement in these steps, both due to imperfect technique and as a result of unintentional mistakes, increases the likelihood of bias and increased variability unless adequate measures are included in the method (7). Fully automated impactors have been developed to reduce operator-related error $(15,16)$, however they are expensive $(>\$ 1 \mathrm{M})$, complex, and resource-intensive to develop and validate because a separate method is needed for each individual product. The future may, therefore, be with a lower-cost semi-automated approach, such as the "Service-Head" technology proposed by Miller et al. (17) in which operator involvement is reduced. As a means of controlling automation costs with the Next Generation Pharmaceutical Impactor (NGI), they proposed simplifying the liquid handling procedures involved in API recovery by making liquid streams required for API dissolution the primary motion of the process, and minimizing the physical motion of the impactor components. Other aids that speed up the process of impactor measurements in a semiautomated way can be an effective and low-cost solution for most users (so-called aided technology). Equipment that enables stage coatings to be applied in a reproducible manner or assists in the reproducible recovery of API from individual stages, are examples of this relatively simple approach to the problem of reducing operator-related error. Initial findings with one such system indicated both excellent API recovery (ca. 99\% of label claim), together with greatly reduced cycle times compared with manual Andersen Cascade Impactor (ACI) (18). Other steps in the direction of increased throughput and reduced human involvement include use of single-stage impactors or liquid impingers (19-21). In addition, an older twin-impinger apparatus is still an effective device for rapid screening studies although it does not provide high size resolution (22). Despite these alternatives and recent developments, the conventional eight-stage ACI (apparatus 1 in the US Pharmacopeia Chapter <601>, 23) and other manual systems based on highly laborious methods are likely to remain in use by most organizations for the foreseeable future especially for regulatory purposes (24). People-related variability can, therefore, be expected to continue to be a significant contributing factor to the overall CI test outcomes. A further detailed discussion of some of the man-related factors is presented below.

\section{Impactor Assembly}

Operator training in impactor assembly is crucial, in particular with stack designs such as the ACI, due to the need to assure correct stage order (7) as well as ensuring that there is a proper seal between impactor stages. A gantry type system could be used to ensure the stack is properly aligned. Proper sealing could be checked via leak tests and/or pressure-drop tests, or by including a differential mass flowmeter at the throat and the impactor exit. A checklist may be a helpful aid, particularly for an inexperienced operator (7). However, even with proper training, occasional errors in the assembly of ACI-type impactors are likely, given the complexity of the process, leading to "failed" CI tests. For example, incorrect ordering (switching) of CI stages or misalignment of collection plates in an ACI are inadvertent errors that may happen even to a well-trained operator (7). A complete set of cups can be fixed permanently into a carrier that makes the process of loading and unloading the impactor more efficient. For an NGI, the stage order is fixed due to the integral nature of the impactor body (25).

\section{Inhaler Handling and Sample Introduction}

Proper training in sample collection is critical because of a wide variety of different test methods, including variations in inhaler handling and introduction of the sample to the CI (26). Even with proper training, individual differences between operators (e.g., inhaler shaking frequency and intensity, delay between shaking and actuation, alignment of inhaler to ACI, actuation) may go unnoticed but may result in different systematic biases (e.g., dose-through-use trends) and also may contribute to the seemingly random variability when results are compared from several operators or even from the same operator on different occasions. For example, a different rate of actuating an MDI may lead to different cooling of the canister (27) and, therefore, different evaporation behavior of the propellant, leading to variations in measured APSD, even when the inhaler units are identical. Using a bench timer may help standardize this aspect of the method and minimize the associated variability; automation of some of these steps may further reduce this type of variability (27).

\section{HPLC Standard Preparation}

When HPLC is used for API assay, an analyst might introduce an "individual" bias into APSD results by preparing the HPLC standard at the upper or lower limit of the predefined range, so that all recoveries from the CI stages would either be slightly over- or under-estimated.

\section{Sample Collection and HPLC Analysis}

The procedure for API recovery from the CI and subsequent analysis offers multiple opportunities for errors including loss of deposited API on disassembly of the impactor prior to API recovery. Therefore, careful handling of the stack and API-coated plates as well as a robust technique for recovering API material deposited on CI surfaces are critical, as is proper organization of samples to 
prevent wrong dilutions and incorrect HPLC vial filling. Pipetting contributes to random variations (more careful pipetting leading to a complete discharge, less careful leading to some drops left over in the pipette), therefore, automated pipettes should be considered. Another potential source of variability is due to the use of different volumes for stage dissolution or for dilution of recovered samples, e.g., in order to obtain the same concentration for all API stage-by-stage samples to be quantified using LC analysis. Potential errors and increased variability should be weighed against potential benefits of this procedure.

Evaporation of solvent during sample recovery will give an overestimation of the API amount and its effects should be minimized, e.g., by inclusion of an internal chemical standard in the solvent used for recovery. If the HPLC method has good linearity, it is also possible to dispense the same volume of internal standard solution to each impactor stage/cup/ throat and to the standard used for API quantitation. In this case, the sample solution is transferred directly to the LC vial and the exact volume transferred is of no importance. The exact concentration of the internal standard solution is not critical as long as the internal standard dispenser is repeatable and the same dispenser is used for both samples and standard solutions.

\section{Stress and Fatigue}

In complex operations, such as CI measurements, stress and fatigue always deteriorate human performance, leading to increased incidence of errors (7). These adverse influences can be minimized by limiting the number of samples to be analyzed per day.

Certain mechanical aids and procedural steps could be introduced to counteract potential operator-related errors. For example, to prevent delivery of an incorrect number of doses to the impactor, use of "counters" linked to activation of the airflow valve may be considered for DPIs; or, the weighing of MDIs could be used before and after the CI test to verify the correct number of doses actuated. As another example, to enable detection of a mix-up of vials from different stages during HPLC analysis, for uni-modal and log-normal APSD distributions, plotting log-probability vs log-particle-size and calculating the regression coefficient could be used as a system suitability tool. Furthermore, mixed-up dilutions could be avoided by using the same amount of solvent for each impactor stage; or by eliminating the dilution step altogether if the detection sensitivity and linearity allow.

\section{Bias Introduced by Experienced Operator}

Even well-trained experienced operators can develop habits that lead to repetitive mistakes in technique. Such errors can often be small but taken together they can accumulate so that the rate of measurement failures increases above typical. Biases from individual operators could be detected through use of control charts (e.g., mass of API reported on key stages tracked as a function of time), and upon further investigation they might be traced to one of the errors listed above. Control charts are particularly useful with repeated testing of established products.

\section{Machine (CI System)}

An APSD measurement is never "absolute" but depends on the technique (28-31), instrument calibration (1), its intrinsic size resolution, as well as the dynamic nature of the aerosol cloud as it interacts with the environment as it enters and passes through the CI (e.g., evaporation kinetics, hygroscopic growth). Therefore, the outcome of an APSD measurement should always be reported along with the specific instrument and technique used. As such, variations of APSD across different techniques are not considered in this article as part of method variability. However, even within the same technique and same instrument type, there is variability due to the factors which may vary with each physical specimen of a given impactor type, and with the conditions at each particular instance of measurement. Compared to other types of analytical measurement, a CI does not have a reference standard, such as a standardized polydisperse aerosol, with which to validate the method, and in that sense every CI measurement is unique. This makes CI data prone to bias and increased variability. Even with the method optimized, unnoticed biases and variability may remain, making it difficult to comply with an a priori specification which disregards data from that particular impactor and method. Careful method development work should try to identify and counteract all major sources of imprecision and bias. Many sources of impactor-related variability have been described in the literature, as summarized below.

In this section, the category "impactor" includes all associated equipment, e.g., the pump creating the airflow and the connecting vacuum tubing. Environmental factors (temperature, humidity) are also included here in as much as they affect the impactor performance. (The effect of such factors on the product is not considered here.) Some of the impactor parameters affecting CI method variability could be "fixed" within a given method (e.g., impactor design, dead volume, pump) and are discussed towards the end of this section. Other parameters may change with time and/or with each instance of measurement (e.g., surface quality, jet dimensions, air leakage, coating thickness) and are discussed first.

\section{Jet Dimensions, Stage Cut-offs, Calibration and Mensuration}

Translation of CI deposition data into a particle size distribution depends on the stage "cut-off" sizes (effective cut-off diameters (ECDs) (2,32). Stage ECDs are determined principally by the actual sizes (diameters) of the nozzles for each stage in a given impactor (33). Several studies have shown, however, that stages of so-called identical CIs have often slightly different nozzle sizes, due either to manufacturing variations or caused by wear, corrosion or accumulation of debris. Partial plugging, wear and corrosion will change the actual size of the nozzles and ECD. These effects result in shifts of API mass between stages (34-36) and, therefore, in bias and increased variability of APSD measurements, especially when data from several impactors are used together (34).

To determine actual ECDs for a given CI, impactor calibration is undertaken with particle standards of known size which is ultimately traceable to the international standard of length (37). Calibration of impactors, however, is a highly 
laborious and expensive process (2) and it is not practically feasible to use it routinely to monitor the performance of an impactor. Therefore, the application of stage mensuration as recommended in the pharmacopeial methods (23) (using optical imaging techniques to measure the diameter or area occupied by each nozzle) as a means to assess effective stage nozzle diameters, continues to be used and investigated as the technique of choice for CI performance verification $(32,38$ 42). Errors in mensuration using stop-go pins have been reported (43), although these pins could be useful for cleaning impactor jets. Recently, the determination of pressure-drop across each stage, which may be performed before every APSD measurement, has been proposed as a surrogate for stage mensuration (44).

\section{Flowrate, Flow Profile, Acceleration and Control}

Stage ECDs are also affected by the flowrate at which the impactor is operated (45). Relatively speaking, nozzle-diametercaused variability in ECD, which is discussed above, is likely to be small in terms of its overall impact on performance, and relatively easily monitored. Flowrate-induced variability, however, is likely to be more significant and less tractable, given the fact that in the pharmacopeial method, the specified flow control is typically no better than $\pm 5 \%$ of the nominal flowrate (23). An alternative to the pharmacopeial method, using a flow-meter calibrated for the entering, rather than exiting, flowrate has been shown to yield similar performance (46). Flowrate variability is an important source of APSD measurement uncertainty, and may vary from one instance of using an impactor to the next instance of using the same impactor.

For MDIs, stage ECDs are determined by the magnitude of the (constant) flowrate achieved during testing; for DPIs, in addition, the flow profile (rise time, acceleration) affects stage ECDs and consequently the measured APSD. The magnitude and direction of these effects depend on the CI design and internal geometry, e.g., the internal dead volume (47-49). It is important to define the vacuum tube length for repeated use of the same impactor type in order to keep the dead volume as constant as possible. The airflow rise time could also be measured as part of the installation checks of a new instrument set-up. Flowrate bias can be minimized if flow-meters are well-maintained, properly calibrated and regularly qualified.

\section{Dead Volume}

In the context of DPI testing, the magnitude of the internal dead volume (open space within the assembled CI, including accessories such as the induction port or preseparator, and vacuum tubing) will influence both the time taken for particles to traverse the system and be collected, as well as the rise time for the flowrate to increase to the final value when the flow control solenoid valve is opened to initiate sampling (2). Recently, the dead volumes of the ACI, NGI and multi-stage liquid impinger (MSLI) were accurately measured with and without the various accessories (50). This information provides a useful starting point when selecting a CI for a new product. Bias introduced to APSD measurements by dead volume will be fixed for a given impactor type and configuration, and ideally the internal volume should be as small as possible. However, the tradeoff between potential bias arising from dead volume and other more important constraints, such as ease of (semi) automation or improved aerodynamic size separating characteristics may result in the choice of a system with a higher internal volume.

\section{Air Leakage}

Air leakage into the CI can arise from incorrectly placed or defective seals or misaligned stages. The problem is particularly prevalent with the standard O-rings used with ACIs, which are prone to cracking with repeated use and exposure to solvents. Defective seals are most significant when they occur at stages closest to the impactor exit, where the pressure inside the stage is at its lowest with respect to the surrounding atmosphere. Since the CI operates under suction from its pump, any air leakage will be into the system. It therefore follows that although the APSD may be shifted (in some cases significantly), the magnitude of the displacement being dependent on leak location and size, the mass balance (MB) for the API will likely be unaffected. Additionally, an increase in inter-stage deposition could occur, which could affect MB if such deposition is not included in the analysis (7). Air leaks are detected and prevented through periodic inspection of seals, careful attention to system assembly, and by comparing the flowrate at the impactor inlet with that downstream of the system as a system suitability check. However, even if these checks are made, it is possible for a small leak between stages to go unnoticed. As part of the CI method development, companies may want to study the effect of leaks on their product's APSD through designed experiments, e.g., by introducing controlled leaks through small cuts in O-rings, and observing the changes in APSD resulted from such simulated failures. This information could later be used for root-cause analysis when atypical APSD profiles are observed, in combination with re-assessment of the tested unit to confirm that the failure is not related to the product.

\section{Environmental Conditions, Temperature and Humidity}

Environmental conditions may influence variability of the aerosol as it is generated by the delivery device (and thus contribute to product variability), but they also may influence APSD during the process of measurement. The first type of influence is not considered here because it is product-specific. The second type could be categorized as CI (method)-related variability. Relative humidity will influence triboelectric effects discussed later, and together with temperature it will also affect droplet growth and evaporation throughout the CI train (51-55), especially for aqueous droplets such as those produced by nebulizers (2). Temperature variations may additionally influence volumetric flowrate and thus further affect the CI measurement. Usually, temperature, and to some extent humidity, are monitored and controlled to minimize these effects. The extent of environmental influences and the needed controls depend on the product type (API, carriers, propellants, co-solvents, etc.), as well as on the CI system and its other operating conditions (e.g., the flowrate may affect the rate of particle growth and evapora- 
tion, the CI temperature may affect heat transfer and droplet evaporation).

\section{Triboelectrification and Electrostatic Charge}

Aerosolized particles are known to carry electrostatic charge generated chiefly by triboelectric effects (contact electrification) during aerosol formation from both DPIs (56-61) and MDIs (62-68). Electrostatic charge acquired by the CI components that are non-conducting or electrically insulated, may influence particle collection behavior (69) and may increase variability of the measured APSD and MB. Depending on the product, exploratory studies may be warranted to optimize the method.

\section{Plate Coating, Coating Thickness and Type}

Coating CI plates has been found to reduce particle bounce and re-entrainment, and thus reduce bias and variability of CI measurements (49,70-71). This effect is particularly important for DPI formulations and may also occur with some MDI products (72), although it may be unimportant for nebulizers (73). The appropriate coating material and thickness are product-dependant. It is unknown, however, whether coating prolongs or shortens plate-life by either preventing or causing corrosion. These effects may also be formulation- and materials-dependant. To standardize coating, some automation or use of a tool that ensures a uniform depth of coating across the collection surface could be considered.

\section{Stage Loading and Number of Actuations}

With formulations requiring multiple actuations for CI testing and containing a high mass-loading of particulates, it is important to consider potential accumulation of material on CI stages to the point at which further incoming particles bounce (74). Not only the amount of API mass depositing on stages needs to be low enough to prevent collected particulate becoming re-entrained in the airflow and transferred to stage (s) further in the CI system, but also the weight of excipient (carrier) per stage must be taken into account. In the extreme, excessive actuations may result in stage overloading that could affect the jet-to-plate distance. If the number of actuations used per a single CI test is too large, an apparent shift to finer particle sizes will be observed (72,74-76). Some recent studies suggest that the type of formulation may also play a role (77). These biases should be studied and eliminated in method development, prior to any method validation, by undertaking measurements with progressively increased numbers of actuations.

\section{Surface Properties}

Different roughness of uncoated stage surfaces may influence ballistic behavior of aerosol particles and measured variability. This effect may depend on the formulation, age of the impactor, and cleaning/drying conditions; however, there are no published data in which the effect of surface roughness on APSD has been investigated. ACI vendors and US Pharmacopeia now describe tolerances for surface roughness but not as a requirement.

\section{Cleaning/Drying}

Proper cleaning/drying of impactor surfaces keeps them as close to their specifications as possible. Variations in the cleaning/drying procedures may affect variability of ASPD measurements. This is obvious from the standpoint of minimizing corrosion (long-term) and having unobstructed jets prior to each measurement (short-term), although there are no published data systematically studying the effect of cleaning/drying regimen on APSD results. Appropriate cleaning/drying procedures should be determined during method development.

\section{Internal (Wall) Losses}

Internal losses of API to the non-collection surfaces within a CI (wall losses) may be an important source of error because of the non-linear association between their magnitude and particle aerodynamic size. Calibration data for the ACI, in which such losses were investigated systematically, indicate a strong association between internal losses and particle aerodynamic size, with increased nonideal deposition particularly evident with particles $>5 \mu \mathrm{m}$ aerodynamic diameter (78). Losses in the pre-separator supplied with this impactor were especially significant. Wall losses should be determined during method development, and be $<5 \%$ of delivered mass; otherwise the material from the entire stage including jets and walls (and not only the impaction plate) should be collected and added to the total dose recovered from the CI (23). Wall losses may depend on the product and method (e.g., coating type, number of actuations).

\section{Induction Port}

The induction port (IP) is important for MDI testing due to the need to capture the ballistic component separately from the fraction that is intended to penetrate beyond the oropharynx into the lower respiratory tract (79). The IP presents the first impaction surface for the moving aerosol once it has left the inhaler (and any add-on device), and therefore, it strongly affects the proportion of the emitted mass entering the impactor. For this reason, the use of adapters to align the MDI actuator with the IP are important for ensuring correct angle of entry of plume. With MDIs, the aerosol plume moves faster than airflow in an IP, resulting in limited time for evaporation and for the reduction in emitted droplet sizes. Furthermore, for any given design of IP, deviations from nominal dimensions and associated tolerances will influence APSD measurements (80-83). Consideration should also be given to the IP surface roughness as this may influence the variability. For nebulizers, an IP is unnecessary because of the absence of the ballistic fraction in nebulizer-produced aerosols.

\section{Pump}

Different pumps may produce different time-dependent flowrate profiles, which are important for DPI testing. The pump-capacity-related variability is minimized by operating the impactor with the flow set by means of a critical orifice (23) and by measuring the critical flow $(\mathrm{P} 3 / \mathrm{P} 2<0.5$, where $\mathrm{P} 2$ and $\mathrm{P} 3$ 
are pressures upstream and downstream of the flow-control valve) $(23,84)$. Because pumps heat up and lose efficiency over time if left running, flowrate setting/verification and APSD measurement should be conducted as quickly as possible.

\section{Impactor Design}

The design of an impactor is another fixed contributor to the overall method variability. The ideal impactor will have stage collection efficiency-versus-particle size curves that are each a step function at the ECD for that stage (2). In practice, this ideal is never achieved, and the collection-efficiency curves of real impactors are sigmoidal in shape, often with slight asymmetry that is associated with non-inertial behavior (i.e., ultra-Stokesian drag and gravitational settling) (85-86). The square root of the ratio of the sizes that correspond to the 84.1st and 15.9th percentiles of these curves has been defined as a quantitative measure of size discrimination, by analogy with the definition of geometric standard deviation as the measure of spread of a cumulative size distribution function. In a well-designed impactor such as the NGI, this value should be close to or $<1.2$ (1.0 represents the ideal step function) (87-88). As well as affecting its size-discriminating capability, the CI design influences the internal airflow characteristics and, therefore, the magnitude of non-ideal deposition on internal surfaces, and in turn, therefore the variability and bias of CI results.

\section{Measurement (Analysis)}

To assay API deposited on CI stages, HPLC/spectrophotometry may be employed, which is a well-characterized and easily standardized technique. Nevertheless, this is yet another area contributing to both random and systematic uncertainty of CI measurements, leading to reduced precision and accuracy of CI results. For example, one of the difficulties of using assay as part of CI testing may be poor chromophore properties of the API and/or low amounts of the API to be assayed, often near the limit of detection (LOD) or limit of quantitation (LOQ) of a given method. Another difficulty is the high number of dilutions and wide ranges of concentrations (e.g., from LOQ for the lowest strength tested to $150 \%$ of the highest deposition for the highest strength tested) necessary for a single CI experiment. The number of actuations used per determination might need to be adjusted to enable an accurate and precise detection method. Additionally, there may be product-specific complications contributing to the overall method variability, for example use of highly volatile solvents (which may be necessary for certain APIs), leading to erratic results due to solvent evaporation during pipetting. Careful method development, involving iterative evaluation and optimization, may help minimize variabilities due to analysis.

\section{Material (Product)}

The APSD of the aerosol delivered by an inhaler or nebulizer may have an inherent variability truly attributable to the product, and there are a number of factors potentially influencing this true APSD variability [e.g., product orienta- tion during storage, formulation interaction with excipients, interaction with components of container closer system, sensitivity to moisture, product age, temperature and humidity during storage, tolerances of device components, static electricity effects during de-aggregation of some DPI formulations, static electricity due to interaction of MDI aerosols with non-conducting elastomers used in MDI valve manufacture, (89-91), etc.]. All such factors should be studied during product development and are not considered here in detail because they are specific to the product rather than the CI method, which is the focus of this review.

In addition, however, certain product characteristics may make the method more variable. For example, suspensions may by nature produce more variable aerosols than homogeneous solutions, arising from the intrinsic tendency for particle segregation in suspensions (product variability). In addition, for suspensions, the timing between shaking and actuation (which is part of the "method") may influence the measured APSD variability. Similarly, for propellant-based MDIs, the rate of actuation (part of the "method") and accompanying drop in the MDI temperature may affect the measured APSD and consequently its variability.

Products combining more than one API may present special challenges for developing a precise and accurate CI method. For example, one of the APIs could be a strong chromophore and the other a weak one, or one of the APIs might reach the limit for stage loading and the other be at the LOD level within the same number of actuations, or one could be a hydrophilic compound and the other a hydrophobic one.

Furthermore, components of variability may arise due to interactions (interdependences) between factors (Material, Man, Machine, and Analysis), e.g., incorrect device cleaning may induce particle growth, giving rise to a man-material interaction component of variability.

Specifically designed experiments could be conducted to separate variability components. The total mass collected from a CI (mass balance) may be used more routinely as a diagnostic tool to distinguish between a method-related and product-related abnormality when deviating APSD results are observed $(7,92)$.

\section{Interrelationships between Factors}

The Ishikawa diagram is a useful tool in visualization of potential root-causes of APSD variability (Fig. 1). However, as already mentioned above, there may be many interactions between the factors, whereby the influence of one factor depends on the presence and magnitude of another or several other factors. For example, from general physico-chemical principles one can expect that the effect of static electricity on the CI measurement could be stronger or weaker depending on the air humidity, type of formulation and CI construction materials. There could also be less obvious interactions present (e.g., factors related to the specific handling technique of the CI operator, depending on the ambient temperature or day or season). Interaction effects cannot be easily depicted in an Ishikawa diagram but they should be studied using designed experiments so that the CI method could be optimized and appropriately controlled.

From Fig. 1 one may also notice that some factors appear more than once, i.e. they affect more than one category. For 
example, the number of actuations may conceivably affect the APSD uncertainty through man (e.g., due to repetitive stress or differences in delay times when firing multiple actuations), as well as through Machine (e.g., due to stage overload and particle bouncing), as well as through measurement (e.g., due to the limit of detection when the number of actuations is small), as well as through material (e.g., due to suspension resettling time). Method developers should be aware of this complexity when optimizing a given CI method or conducting an investigation of deviating results.

\section{Method Optimized: What Next?}

Even with the best possible method, some variability in CI results will remain, and specific experiments should be carried out to identify and quantify the contribution of major factors of interest. The present report is intended to aid in the identification of the most important factors. As a next step, a sponsor should consider designing and conducting an experiment to quantify the contribution of major factors, by consulting specialized literature on design of experiments (93-100). Such careful method development work will increase sponsor's understanding of the method capability and variability, which in turn could help establish appropriate quality control programs and acceptance criteria.

\section{CONCLUSIONS}

In order to produce pharmaceutical products of high quality with maximum efficiency in line with the modern regulatory quality-by-design concepts, a sponsor must achieve and demonstrate an understanding and optimal design of the product, test methods, and manufacturing process. The current review could guide sponsors' efforts to optimize capability and variability of the CI test method, which is frequently employed for one of the key quality measures of inhaled products. Judging from published literature, of the four major categories of factors affecting CI measurements, impactor-related factors have been studied the most. Much less published information, especially quantitative data, exists about operator-derived influences, as well as the contribution of API assay methodology and product-related causes to the outcome of a CI test; specifically designed experiments by individual sponsors or collaborative groups could therefore significantly help advance our knowledge in these areas.

\section{ACKNOWLEDGEMENT}

Authors thank the IPAC-RS Board of Directors for encouraging this work and providing feedback on the draft manuscript.

\section{REFERENCES}

1. J. P. Mitchell, and M. W. Nagel. Particle size analysis of aerosols from medicinal inhalers. KONA-Powder and Particle 22:32-65 (2004).

2. J. P. Mitchell, and M. W. Nagel. Cascade impactors for the size characterization of aerosols from medical inhalers: their uses and limitations. J. Aerosol. Med. 16(4):341-377 (2003).
3. J. P. Mitchell, and M. Tservistas. Laser diffractometry and cascade impaction for nebulizer product characterization. Pharmeuropa Scientific Notes 2:49-52 (2006).

4. J. P. Mitchell, M. W. Nagel, S. Nichols, and O. Nerbrink. Laser diffractometry as a technique for the rapid assessment of aerosol particle size from inhalers. J. Aerosol. Med. 19(4):409-433 (2006).

5. W. C. Hinds. Aerosol technology properties, behavior, and measurement of airborne particles, 2nd ed., Wiley, New York, 1999, pp. 134-138.

6. K. Leung, E. Louca, M. Gray, G. Tipples, and A. L. Coates. Use of the next generation pharmaceutical impactor for particle size distribution measurements of live viral aerosol vaccines. J. Aerosol. Med. 18(4):414-426 (2005).

7. D. Christopher, P. Curry, B. Doub, K. Furnkranz, M. Lavery, K. Lin, S. Lyapustina, J. Mitchell, B. Rogers, H. Strickland, T. Tougas, Y. Tsong, and B. Wyka. Considerations for the development and practice of cascade impaction testing including a mass balance failure investigation tree. J. Aerosol. Med. 16:235-247 (2003).

8. J. Mitchell. Regarding the development and practice of cascade impaction testing, including a mass balance failure investigation tree. J. Aerosol. Med. 16(4):433-433 (2003).

9. International Conference on Harmonisation of Technical Requirements for Registration of Pharmaceuticals for Human Use (ICH). Pharmaceutical Development Q8ICH, Geneva, 2005. Accessed January 2, 2008 at http://www.ich.org.

10. International Conference on Harmonisation of Technical Requirements for Registration of Pharmaceuticals for Human Use (ICH). Quality Risk Management Q9, ICH, Geneva, 2005. Accessed January 2, 2008 at http://www.ich.org.

11. A. S. Hussain. Pharmaceutical quality by design: improving emphasis on manufacturing science in the 21 st century. FDA Pharmaceutical Inspectorate Conference, 2004. Accessed January 2, 2008 at http://www.fda.gov/cder/OPS/PharmQual.pdf.

12. A. S. Hussain. Engineering a proactive decision system for pharmaceutical quality: integrating science of design, process analytical technology and quality system. Presentation at IFPAC conference, 2005. Accessed January 2, 2008 at http:// www.fda.gov/cder/OPS/hussain_1_2005.pdf.

13. A. S. Hussain. Quality by design $(\mathrm{QbD})$-integration of prior knowledge and pharmaceutical development into cmc submission and review. Presentation at AAPS/FDA workshop, 2005. Accessed January 2, 2008 at http://www.aapspharmaceutica. com/workshops/PharmaceuticalQuality100505/hussain.pdf.

14. J. Mitchell, S. Newman, and H.-K. Chan. In Vitro and In Vivo Aspects of Cascade Impactor Tests and Inhaler Performance: A Review. AAPS PharmSciTech. 2007; 8(4): Article 110. DOI 10.1208/pt0804110 (Accessed February 13, 2008 at http://www. aapspharmscitech.org/view.asp?art=pt0804110).

15. M. Smith. Ictus ${ }^{\mathrm{TM}}$ automated impactor particle sizing. Resp. Drug Deliv. 7:451-454 (2000).

16. M. P. Smith. Automation of the next generation impactor-first the Andersen, now the NGI. Drug Deliv. Lungs 11:131-134 (2000).

17. N. C. Miller, D. L. Roberts, and V. A. Marple. The 'service head' approach to automating the next generation pharmaceutical impactor: proof of concept. Resp. Drug Deliv. 8:521-523 (2002).

18. K. Fransson, M. Persson, and M. Svensson. Sample preparation tools for the next generation pharmaceutical impactor. Drug Deliv. Lungs 8:127-130 (2002).

19. H. Lundbäck, and B. Wiktorsson. High throughput inhaler testing I: fine particle dose. Resp. Drug Deliv. 10:467-469 (2006).

20. E. Berg, and O. Svensson. High throughput inhaler testing II: fine particle assessment using internal standard LC methodology. Resp. Drug Deliv. 10:471-474 (2006).

21. M. Svensson. High throughput inhaler testing III: modified aerodynamic particle sizer set-up for DPIs. Resp. Drug Deliv 10:475-478 (2006).

22. G. W. Hallworth, and D. G. Westmoreland. The twin impinger: a simple device for assessing the delivery of drugs from metered dose pressurized aerosol inhalers. J. Pharm. Pharmacol. 39:966972 (1987).

23. United States Pharmacopeial Convention (USP). Chapter 601: aerosols, metered-dose inhalers, and dry powder inhalers. USP30-NF25:220-240 (2007). 
24. US Food and Drug Administration (FDA), CDER. Draft guidance for industry metered dose inhaler (MDI) and dry powder inhaler (DPI) drug products chemistry, manufacturing, and controls documentation, FDA, 1998. Accessed January 2, 2008 at http://www.fda.gov/cder/guidance/2180dft.pdf.

25. V. A. Marple, D. L. Roberts, F. J. Romay, N. C. Miller, K. G. Truman, M. Van Oort, B. Olsson, M. J. Holroyd, J. P. Mitchell, and D. Hochrainer. Next generation pharmaceutical impactor. Part 1: Design. J. Aerosol. Med. 16:283-299 (2003).

26. T. S. Purewal. Test methods for inhalers to check performance under normal use and unintentional misuse conditions. Drug Deliv. Lungs 12:92-98 (2001).

27. E. Stewart, J. Holt, C. Fitzgerald, P. Bell, and J. Popow. Impact of using an automated shake-fire system on the shot weight and dose content uniformity of an HFA metered dose inhaler. Resp. Drug Deliv. 102:581-583 (2006).

28. P. M. Holzner, and B. W. Muller. Particle size determination of metered dose inhalers with inertial separation methods: apparatus A and B (BP), four stage impinger and Andersen Mark II cascade impactor. Int. J. Pharm. 116:11-18 (1995).

29. J. P. Mitchell, M. W. Nagel, K. J. Wiersema, and C. C. Doyle. Aerodynamic particle size analysis of aerosols from pressurized metered dose inhalers: comparison of Andersen 8-stage cascade impactor, next generation pharmaceutical impactor, and model 3321 aerodynamic particle sizer aerosol spectrometer. AAPS PharmSciTech. 4(4):article 54 (2003). Accessed January 2, 2008 at http://www.aapspharmscitech.org/view.asp?art=pt040454\& pdf=yes.

30. M. Taki, X. M. Zeng, C. Marriott, and G. Martin. Comparison of deposition profiles of drugs from a combination dry powder inhaler using the Andersen cascade impactor (ACI), multistage liquid impinger (MSLI) and next generation impactor (NGI). Resp. Drug Deliv. 10(2):659-662 (2006).

31. J. Jozwiakowski, X. Lor, S. Paulson, and D. Schultz. Comparison of Andersen cascade impactor and next generation impactor performance of beclomethasone pMDIs with oligolactic acid. Resp. Drug Deliv. 10(2):357-359 (2006).

32. J. P. Mitchell, and C. Dunbar. Analysis of cascade impactor mass distributions. J. Aerosol. Med. 18(4):439-451 (2005).

33. D. L. Roberts, and F. J. Romay. Relationship of stage mensuration data to the performance of new and used cascade impactors. J. Aerosol. Med. 18:396-413 (2005).

34. S. W. Stein, and B. A. Olson. Variability in size distribution measurements obtained using multiple Andersen mark II cascade impactors. Pharm. Res. 14:1718-1725 (1997). Accessed 2, 2008 at http://www.aapspharmaceutica.com/search/view.asp? ID $=14941$

35. S. W. Stein. Size distribution measurements of metered dose inhalers using Andersen mark II cascade impactors. Int. J. Pharm. 186:43-52 (1999).

36. N. Kadrichu, N. Rao, G. Sluggett, B. Fong, G. Jones, T. Perrone, S. Seshadri, P. Shao, G. Williams, J. Zhang, and D. Bennett. Sensitivity of Andersen cascade impactor response to stage nozzle dimensions. Resp. Drug Deliv. 9:561-564 (2004).

37. J. P. Mitchell. Particle standards: their development and application. KONA-Powder and Particle 18:41-59 (2000).

38. K. Milhomme, C. Dunbar, C. Lavarreda, D. Roberts, and F. Romay. Measuring changes in the effective jet diameter of cascade impactor stages with the flow resistance monitor. Resp. Drug Deliv. 10(2):405-407 (2006).

39. D. L. Roberts, C. Lavarreda, and K. Milhomme. Managing impactor quality with measurements of flow resistance and effective diameter. Drug Deliv. Lungs 18:243-246 (2006).

40. C. M. Shelton. Comparison of optical systems for mensuration of eight-stage cascade impactors. Drug Deliv. Lungs 15:133-136 (2004).

41. C. Shelton, J. P. Mitchell, D. L. Roberts. Cascade impactor mensuration: deciding the important specifications. In: R. N. Dalby, P. R. Byron, J. Peart, and J. D. Suman (eds.), Respiratory Drug Delivery-Europe, Davis Horwood International, River Grove, IL, 2005, pp. 29-34.

42. C. M. Shelton, and D. L. Roberts. Application of flow resistance measurements to cascade impactor quality control. Drug Deliv. Lungs 16:221-224 (2005).
43. M. Svensson, G. Pettersson, and L. Asking. Mensuration and cleaning of the jets in Andersen cascade impactors. Pharm. Res. 22(1):161-165 (2005).

44. K. Milhomme, C. Dunbar, C. Lavarreda, D. Roberts, and F. Romay. Measuring changes in the effective jet diameter of cascade impactor stages with the flow resistance monitor. Resp. Drug Deliv. 10:405-407 (2006).

45. V. A. Marple, K. L. Rubow, and B. A. Olson. Inertial, gravitational, centrifugal, and thermal collection techniques. In: P. A. Baron and K. Willeke(eds.), Aerosol Measurement: Principles, Techniques and Applications, 2nd ed., Wiley, New York, 2001, pp. 229-260.

46. B. Olsson, and L. Asking. Methods of setting and measuring flowrates in pharmaceutical impactor experiments. Drug Deliv. Lungs 13:205-208 (2002).

47. B. Wiktorsson, and L. Asking. Comparison between flowmeters used to set flows in pharmaceutical inhaler testing. Drug Deliv. Lungs 13:168-171 (2002).

48. M. Van Oort, B. Downey, and W. Roberts. Verification of operating the Andersen cascade impactor at different flowrates. Pharm. Forum 22(2):2211-2215 (1996).

49. W. C. Hinds. Aerosol technology properties, behavior, and measurement of airborne particles. 2nd ed., Wiley, New York, 1999, pp. 128-134.

50. M. Copley, M. Smurthwaite, D. L. Roberts, and J. P. Mitchell. Revised internal volumes of cascade impactors for those provided by Mitchell and Nagel. J. Aerosol. Med. 18:364-366 (2005).

51. S. W. Stein, and P. B. Myrdal. The relative influence of atomization and evaporation on metered dose inhaler drug delivery efficiency. Aerosol. Sci. Technol. 40:335-347 (2006).

52. C. Peng, A. Chow, and C. K. Chan. Study of the hygroscopic properties of selected pharmaceutical aerosols using single particle levitation. Pharm. Res. 17(9):1104-1109 (2000).

53. W. H. Finlay. Estimating the type of hygroscopic behavior exhibited by aqueous droplets. J. Aerosol. Med. 11:221-229 (1998).

54. P. R. Byron, S. S. Davis, M. D. Bubb, and P. Cooper. Pharmaceutical implications of particle growth at high relative humidities. Pesticide Sci. 8(5):521-526 (1977).

55. A. R. Martin and W. H. Finlay. Effect of humidity on size distributions of MDI particles exiting a mechanical ventilation holding chamber. Proceedings of International Conference on MEMS, NANO and Smart Systems, 2004. ICMENS, 2004, pp. $280-283$.

56. P. R. Byron, J. Peart, and J. N. Staniforth. Aerosol electrostatics I: Properties of fine powders before and after aerosolization by dry powder inhalers. Pharm. Res. 14:698-705 (1997).

57. M. Murtomaa, V. Mellin, P. Harjunen, T. Lankinen, E. Laine, and V. P. Lehto. Effect of particle morphology on the triboelectrification in dry powder inhalers. Int. J. Pharm. 282(12):107-114 (2004).

58. P. A. Carter, O. E. Cassidy, G. Rowley, and D. R. Merrifield. Triboelectrification of fractionated crystalline and spray dried lactose. Pharm. Pharmacol. Commun. 4:111-115 (1997).

59. P. A. Carter, G. Rowley, and N. J. McEntee. An investigation of experimental variables during triboelectrification studies on powders. J. Pharm. Pharmacol. 49(S4):23 (1997).

60. M. Murtomaa, S. Strengella, E. Lainea, and A. Baileyb. Measurement of electrostatic charge of an aerosol using a grid-probe. J. Electrost. 58(3-4):197-207 (2003).

61. J.-C. Ramirez-Dorronsoro, R. B. Jacko, and D. O. Kildsig. Chargeability measurements of selected pharmaceutical dry powders to assess their electrostatic charge control capabilities. AAPS PharmSciTech. 7(4):article 103 (2006). Accessed January 2, 2008 at http://www.aapspharmscitech.org/view.asp? art $=$ pt0704103.

62. P. C. L. Kwok, W. Glover, and H. K. Chan. Electrostatic charge characteristics of aerosols produced from metered dose inhalers. J. Pharm. Sci. 94(12):2789-2799 (2005).

63. W. Glover, P. Kwok, and H. K. Chan. Electrostatic charges in metered dose inhalers. Resp. Drug Deliv. 9:829-832 (2004).

64. W. Glover, and H. K. Chan. Electrostatic charge characterization of pharmaceutical aerosols using electrical low-pressure impaction (ELPI). J. Aerosol. Sci. 35:755-764 (2004). 
65. M. Crampton, R. Kinnersley, and J. Ayres. Sub-micrometer particle production by pressurized metered dose inhalers. $J$. Aerosol. Med. 17(1):33-42 (2004).

66. W. Glover, and H. K. Chan. Electrostatic charge characterization of pharmaceutical aerosols. Resp. Drug. Deliv 9:825-826 (2004).

67. P. Kwok, and H. K. Chan. Measurement of electrostatic charge of nebulised aqueous droplets with the electrical low pressure impactor. Resp. Drug Deliv. 9:833-836 (2004).

68. J. Peart, C. Magyar, and P. R. Byron. Aerosol electrostaticsmetered dose inhalers (MDIs): Reformulation and device design issues. Resp. Drug Deliv. 6:227-233 (1998).

69. K. D. Horton, and J. P. Mitchell. The calibration of a California measurements PC-2 quartz crystal cascade impactor. J. Aerosol. Sci. 23:505-524 (1992).

70. M. M. Nasr, D. L. Ross, and N. Miller. Effect of drug loading and plate coating on the particle size distribution of a commercial albuterol metered dose inhaler (MDI) determined using the Andersen and Marple-Miller cascade impactor. Pharm. Res. 14(10):1437-1443 (1997).

71. J. Mitchell, and European Pharmaceutical Aerosol Group (EPAG). Practices of coating collection surfaces of cascade impactors: a survey of members of EPAG. Drug Deliv. Lungs 14:75-78 (2003).

72. A. Kamiya, M. Sakagami, M. Hindle, and P. R. Byron. Locating particle bounce in the next generation impactor (NGI). Resp. Drug Deliv. 9:869-871 (2004).

73. E. Berg, J. O. Svensson, and L. Asking. Determination of nebulizer droplet size distribution: a method based on impactor refrigeration. J. Aerosol. Med 20(2):97-104 (2007).

74. A. Kamiya, M. Sakagami, M. Hindle, and P. R. Byron. Aerodynamic sizing of metered dose inhalers: an evaluation of the Andersen and next generation pharmaceutical impactors and their USP methods. J. Pharm. Sci. 93(7):1828-1837 (2004).

75. M. M. Nasr, and J. F. Allgire. Loading effect on particle size measurements by inertial sampling of albuterol metered dose inhalers. Pharm. Res. 12(11):1677-1681 (1995).

76. M. R. Feddah, and N. M. Davies. Influence of single versus multiple actuations on the particle size distribution of beclomethasone dipropionate metered-dose inhalers. J. Pharm. Pharmacol. 55:1055-1061 (2003).

77. C. Merrin, S. Lee, M. Needham, and F. Chambers. Evaluation of NGI performance with high dose pMDIs. Drug Deliv. Lungs 14:184-187 (2003).

78. J. P. Mitchell, P. A. Costa, and S. Waters. An assessment of an Andersen Mark-II cascade impactor. J. Aerosol. Sci. 19(2):213221 (1987).

79. M. Dolovich, and R. Rhem. Impact of oropharyngeal deposition on inhaled dose. J. Aerosol. Med. 11(1):S112-S121 (1998).

80. D. Harris, S. Chaudhry, I. Chaudry, S. Li, J. Sequeira, and B. Wyka. Influence of entry-port design on drug deposition in cascade-impactor from metered-dose inhalers. AAPS Annual Meeting. AAPS Poster Session, 1996.

81. M. Van Oort, and B. Downey. Cascade impaction of MDIs and DPIs: proposal of induction port, inlet cone, and preseparator lid designs for inclusion in general chapter <601>. Pharm. Forum. 22(2):2204-2210 (1996)

82. M. Van Oort, and K. Truman. What is a respirable dose? $J$. Aerosol. Med. 11(1):S89-S96 (1998).

83. M. Van Oort, R. Gollmar, and R. Bohinski. Effects of sampling chamber volume and geometry on aerodynamic size distribu- tions of metered-dose inhalation aerosols measured with the Andersen cascade, impactor. Pharm. Res. 11:604 (1994).

84. European Pharmacopeia. Section 2.9.18-Preparations for inhalation: aerodynamic assessment of fine particles. European Pharmacopeia, 5th ed. 1st supplement, Council of Europe, 67075 Strasbourg, France, 2005, pp. 2799-2811.

85. D. J. Rader, and V. A. Marple. Effect of ultra-Stokesian drag and particle interception on impactor characteristics. Aerosol. Sci. Technol. 4:141-156 (1985).

86. D. J. Rader, and V. A. Marple. Effect of gravitational forces on the calculation of impactor efficiency curves. In B. Y. H. Liu, D. Y. H. Pui, and H. J. Fissan (eds.), Aerosols, Elsevier, New York, 1984, pp. 123-126.

87. V. A. Marple, B. A. Olson, K. Santhanakrishnan, J. P. Mitchell, S. C. Murray, and B. L. Hudson- Curtis. Next generation pharmaceutical impactor (A new impactor for pharmaceutical inhaler testing)_Part 2: Archival calibration. J. Aerosol. Med. 16:301-324 (2003)

88. V. A. Marple, B. A. Olson, K. Santhanakrishnan, J. P. Mitchell, S. C. Murray, and B. L. Hudson-Curtis. Next generation pharmaceutical impactor (A new impactor for pharmaceutical inhaler testing). Part 3: extension of archival calibration to $15 \mathrm{~L} /$ min. J. Aerosol. Med. 17:335-343 (2004).

89. J. C. Keil, K. Reshima, and J. Peart. Using and interpreting aerosol electrostatic data from the electrical low pressure impactor. Resp. Drug Deliv. 10:267-277 (2006).

90. J. Peart, J. C. Orban, P. McGlynn, M. Redmon, C. M. Sargeant, and P. R. Byron. MDI electrostatics: valve and formulation interactions which really make a difference. Resp. Drug Delivery 8:223-230 (2002).

91. P. R. Byron, J. Peart, and J. N. Staniforth. Aerosol electrostatics I: properties of fine powders before and after aerosolization by dry powder inhalers. Pharm. Res. 14(6):698-705 (1997).

92. H. Bagger-Jörgensen, D. Sandell, H. Lundbäck, and M. Sundahl. Effect of inherent variability of inhalation products on impactor mass balance limits. J. Aerosol Med 18(4):367-378 (2005)

93. G. E. Box, W. G. Hunter, J. S. Hunter, and W. G. Hunter Statistics for experimenters: design, innovation, and discovery, 2nd ed, Wiley, Hoboken, NJ, 2005.

94. H. Chernoff. Sequential analysis and optimal design, 2nd ed. (CBMS-NSF Regional Conference Series in Applied Mathematics). Society for Industrial \& Applied Mathematics, Philadelphia, PA, 1987

95. L. Eriksson, E. Johansson, N. Kettaneh-Wold, C. Wikström, and S. Wold. Design of experiments: principles and applications, Umetrics, Kinnelon, NJ, 2001.

96. R. Mead. Design of experiments: statistical principles for practical applications, Cambridge University Press, Cambridge, UK, 1990.

97. C. K. Bayne, and I. B. Rubin. Practical experimental designs and optimization methods for chemists, $\mathrm{VCH}$, Deerfield Beach, FL, 1986.

98. G. E. P. Box, and N. R. Draper. Empirical model—building and response surfaces, Wiley, New York, 1987.

99. E. Morgan. Chemometrics: experimental design, Wiley, New York, 1991

100. B. Wyka, T. Tougas, J. Mitchell, H. Strickland, D. Christopher, and S. Lyapustina. Comparison of two approaches for treating cascade impaction mass balance measurements. J. Aerosol. Med. 20(3):236-256 (2007) 